\title{
POLÍTICAS PÚBLICAS NA GESTÃO DE RISCO DE DESASTRES: UM OLHAR PARA ALÉM DA RESPOSTA À EMERGÊNCIA NO SISTEMA ÚNICO DE SAÚDE
}

\author{
Eliane Lima e Silva \\ Pesquisadora Assistente \\ Laboratório de Geografia, Ambiente e Saúde - LAGAS \\ Departamento de Geografia - GEA \\ Universidade de Brasília - UnB \\ elianelima26@gmail.com
}

\section{INTRODUÇÃO}

Desastres são eventos cada vez mais frequentes que se apresentam como uma ameaça à vida das pessoas, provocando impactos diretos e indiretos, mensuráveis e imensuráveis, então, falar sobre saúde em situações de desastres é um desafio. Esse tema nos leva a diversas ponderações das quais, considerando o cenário brasileiro, quero convidá-los a refletir sobre:

a) quais os impactos potenciais que os desastres "naturais" podem trazer para a saúde das pessoas e para os serviços de saúde?

b) as políticas públicas envolvidas nessa temática têm contribuído para o estabelecimento de uma agenda de redução do risco de desastres e para a redução do risco que esses desastres representam para a saúde humana?

c) há consonância entre as decisões setoriais na implementação de políticas públicas e as medidas de redução do risco de desastres?

d) há perspectivas sobre a adoção de medidas para a redução do risco atual e futuro que os desastres representam no Brasil?

Todos esses pontos subsidiam uma complexa análise cercada de incertezas. Assim, vale fazer uma reflexão sobre a relação entre desastres e saúde e conhecer a estratégia de gestão de risco de desastres e a preparação e resposta à emergência em saúde pública por desastres no Sistema Único de Saúde, que será o desafio a ser apresentado neste texto.

\section{SAÚDE E AMBIENTE}

Saúde e ambiente estão estreitamente ligados e essa relação define o campo do conhecimento da Saúde Ambiental. No Brasil, essa temática foi subsidiada por discussões no âmbito das Nações Unidas e a preparação para a Eco-92, bem como seus resultados, proporcionaram a ampliação das discussões, tanto acadêmico-científica quanto governamental e, no campo da saúde, a Conferência Pan-Americana sobre Saúde, Ambiente e Desenvolvimento (COPASAD), realizada pela Organização Pan-Americana de Saúde (OPAS) em 1995, intensificou as ações nacionais que resultaram na institucionalização da Vigilância em Saúde Ambiental no Sistema Único de Saúde sob a responsabilidade da Fundação Nacional de Saúde (FUNASA), em 2000, e hoje alocada na Secretaria de Vigilância em Saúde (SVS) (Tambellini \& Câmara, 1998; Brasil, 2002; Barcellos \& Quitério, 2006; Brasil, 2018).

Saúde Ambiental envolve um conjunto de ações no intuito de proporcionar o conhecimento e a detecção de mudanças nos fatores do meio ambiente que interferem na saúde humana. Dentre esses fatores encontram-se as emergências e desastres, tanto naturais quanto tecnológicos, que são objetos de interesse do Subsistema de Vigilância em Saúde Ambiental (SIBSA) (Brasil, 2002; Brasil, 1995; Brasil, 2018).

Recebido em: 13/10/2019

Aceito para publicação em: 11/11/2019

Palestra proferida no IX Simpósio Nacional de Geografia da Saúde, Blumenau, SC, 2019.

página 91 
Políticas públicas na gestão de risco de desastres: um olhar para além da resposta à emergência no Sistema Único de Saúde

A redução de riscos de desastres constitui uma das funções essenciais da saúde pública que envolve, dentre outros aspectos:

a. O desenvolvimento de políticas, o planejamento e a realização de ações de prevenção, mitigação, preparação, resposta e reabilitação para reduzir o impacto dos desastres sobre a saúde pública;

b. Um enfoque integral sobre os danos e a origem de todas ou cada uma das emergências ou desastres possíveis na realidade do país; a participação de todo o sistema de saúde e a mais ampla colaboração intersetorial e interinstitucional na redução do impacto de emergências ou desastres.

Os impactos e prejuízos causados por desastres demonstra a necessidade do estabelecimento de políticas públicas relacionadas à gestão do risco que eles representam e se estabelecem como um desafio a ser superado. A estimativa dos potenciais impactos dos desastres em diversos setores é de suma importância para pesquisadores e acadêmicos, mas principalmente para formuladores de políticas e governamentais (Caruso 2017).

Considerando que a mudança do clima influencia a ocorrência de eventos climáticos extremos aumento do nível do mar, aumentam inundações, pioram as secas e estiagem, exacerbam ondas de calor, tempestades e altera o comportamento de vetores de doenças, dentre outros - e os processo de desenvolvimento, quando não sustentável, amplia as vulnerabilidades, fortalecer a capacidade de análise de situação, o conhecimento do risco e ampliar a capacidade de resposta a emergências e desastres são apontados como necessário desde a década de 70 do século passado e uma das iniciativas precursoras foi a criação do Programa de Preparativos para Situações de Emergência e Resposta a Desastres em Saúde na Organização Pan-Americana da Saúde (Ugarte, Aguilar, and Mauvernay 2018).

Os desastres podem causar impactos diversos sobre a saúde e eles dependem da tipologia do evento, das vulnerabilidades da área atingida e da capacidade de resiliência presente no local. Isso se aplica tanto quando o foco é a comunidade quanto as instituições envolvidas na resposta a esses eventos. Cada desastre tem um comportamento que pode se alterar, mesmo que seja a mesma tipologia, ou seja, uma inundação será diferente a cada ocorrência, mesmo quando atingem o mesmo lugar.

\section{CLASSIFICAÇÃO DE DESASTRES}

Desastres são classificados como o resultado de eventos adversos, naturais ou provocados pelo homem sobre um cenário vulnerável, causando grave perturbação ao funcionamento de uma comunidade ou sociedade envolvendo extensivas perdas e danos humanos, materiais, econômicos ou ambientais, que excede a sua capacidade de lidar com o problema usando meios próprios (BRASIL, 2016).

O Brasil estabeleceu a Classificação e Codificação Brasileiras de Desastres (COBRADE) que está adequada ao que estabelece o Centro para Pesquisa sobre Epidemiologia de Desastres (CRED), da Organização Mundial de Saúde (OMS/ONU) que classificam os desastres quanto à intensidade (níveis I e II), evolução (súbitos ou graduais), origem (naturais ou tecnológicos) e periodicidade (esporádicos ou cíclicos/sazonais) (BRASIL, 2016).

Nessa classificação, os desastres naturais referem-se aqueles causados por processos ou fenômenos naturais enquanto que os tecnológicos são aqueles originados de condições tecnológicas ou industriais, incluindo acidentes, procedimentos perigosos, falhas na infraestrutura ou atividades humanas específicas.

biológicos

- Desastres naturais: geológicos, hidrológicos, meteorológicos, climatológicos e

- Desastres tecnológicos: radioativos, produtos perigosos (químicos), obras civis (rompimento/colapso de barragem).

Ambos podem implicar em perdas humanas ou outros impactos à saúde, danos ao meio ambiente, à 
Políticas públicas na gestão de risco de desastres: um olhar para além da resposta à emergência no Sistema Único de Saúde

propriedade, interrupção dos serviços e distúrbios sociais e econômicos.

A incidência de desastres naturais tem aumentado e o impacto das mudanças climáticas ampliará o risco para milhões de indivíduos, seus lares, suas comunidades e a infraestrutura que os sustenta.

\section{DESASTRES E OS IMPACTOS SOBRE A SAÚDE}

O risco que os desastres podem representar para a saúde varia conforme a tipologia, a frequência e a dimensão dessas ocorrências. Os desastres impactam a saúde de diferentes formas, que pode ser direta ou indireta, e esses efeitos perduram ao longo do tempo, variando de curto a longo prazo. As especificidades locais devem ser consideradas para o fortalecimento da atuação do setor saúde nessa temática, abrangendo, como subsídio para à tomada de decisão, o histórico espaço-temporal da ocorrência dos eventos, bem como as notificações de doenças e agravos à saúde (OPAS, 2015).

A exposição aos desastres pode desencadear uma emergência em saúde pública, que é compreendida como aquelas situações nas quais o emprego urgente de medidas preventivas e de controle e contenção de riscos, danos e agravos à saúde pública são demandados em virtude da ocorrência de situações epidemiológicas, desastres ou desassistência à população, conforme estabelece o Decreto Presidencial, n 7.616, 17 de novembro de 2011 (BRASIL, 2011; 2014). Assim, o fortalecimento da capacidade de atuação do setor saúde em emergência em saúde pública por desastres, sejam eles de origem natural ou tecnológica, é primordial para reduzir o risco à saúde da população e à prestação de serviços.

Esse risco está associado aos potenciais impactos sobre as pessoas, incluindo os profissionais de saúde, e sobre a infraestrutura podendo diminuir a capacidade de atendimento dos sistemas de saúde justamente quando há a possibilidade de ampliação da demanda por esses serviços (FREITAS et al, 2018).

Assim, preparar o setor saúde para atuar em situações de desastres é uma das formas de reduzir os danos por eles provocados e, em um país da dimensão do Brasil, isso se apresenta como um desafio à formulação e implementação de políticas públicas de gestão do risco de desastres no intuito de fortalecer os sistemas de prevenção, preparação e resposta a desastres por meio de abordagens intersetoriais e multidisciplinares.

\section{A GESTÃo de RISCO DE DESASTRES E SAÚdE: OLHAR PARA ALÉM DA RESPOSTA À EMERGÊNCIA}

A resposta a emergências e desastres é parte da rotina do setor saúde. O desafio contínuo é ampliar o foco da resposta para a gestão do risco, a partir de uma abordagem proativa que envolva ações de prevenção e mitigação e que tornem os serviços de saúde mais resilientes com a clareza de que para uma atuação oportuna em emergências e desastres é necessário preparação prévia. Essa é uma das premissas para a gestão do risco de desastres e é apontada como uma estratégia essencial de redução dos danos.

Os impactos causados pelos desastres são de natureza multirrisco e sua dimensão está diretamente relacionada às condições de vulnerabilidades e a capacidade de resiliência da área atingida. Políticas públicas diversas (econômica, social, ambiental, dentre outras) permeiam a gestão de risco de desastres e a articulação entre elas é apontada como uma das formas de maximizar as capacidades instaladas e reduzir a sobreposição de ações em âmbito governamental.

As condições ambientais, o uso e ocupação do solo, as desigualdades sociais e o acesso aos serviços públicos (saúde, moradia, educação, assistência social, etc.), dentre outros, influenciam a situação vulnerabilidade e podem ser a fonte dos fatores que definem o grau de risco à saúde das pessoas.

Nesse contexto, quando se trabalha a gestão de risco de desastres, independente do setor ou da política, deve ter claro que este é um processo que exige uma atuação contínua e que é pautado em iniciativas voltadas para a redução do risco, para o manejo do desastre e para a recuperação dos seus efeitos (Figura 1). 
Figura 1: Processo de gestão do risco de desastres

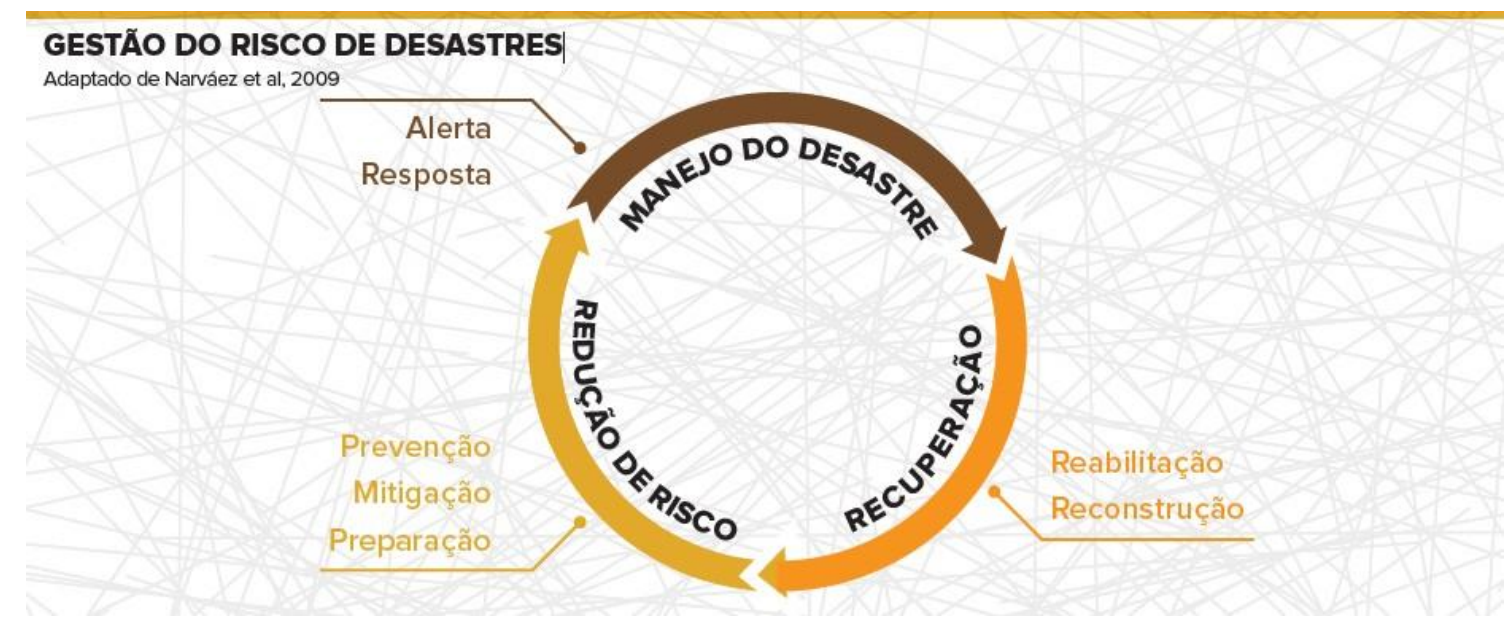

Fonte: Freitas, CM, 2014

A gestão do risco de desastres contempla uma visão multidisciplinar e intersetorial que considera, na análise de situação de saúde, o cenário de risco incluindo as potenciais ameaças ou perigos, as vulnerabilidades e a capacidade de resiliência buscando a identificação de pontos-chave para se antecipar aos eventos e com isso reduzir seus impactos.

\section{DESAFIOS PARA POLÍTICAS PÚBLICAS DE REDUÇÃO DO RISCO}

Dotar os serviços de saúde de capacidades básicas para o gerenciamento de risco de desastres em um país com a dimensão do Brasil é um tanto complexo, mas, sabe-se que quanto mais conhecimento do cenário de risco local, melhor será a atuação das secretarias de saúde, mesmo com as limitações estruturais existentes na maioria delas. O próprio processo de preparação para responder a emergência propicia que a instituição promova a articulação interna e interinstitucional, conheça as capacidades instaladas, identifique as necessidades para uma atuação oportuna e mapeie uma rede de apoio adicional, caso necessário.

O funcionamento das ações de atenção básica e de vigilância em saúde propicia melhor capacidade de intervir nos fatores de riscos de uma comunidade e a torna mais resiliente para superar os potenciais efeitos provocados por desastres. O conhecimento do perfil epidemiológico e da estrutura da rede de atendimento à saúde subsidia a adoção de medidas para propiciar a proteção à saúde das pessoas e a continuidade da prestação dos serviços de saúde, mesmo em situações de desastres.

O investimento na ampliação da capacidade local para atuar em emergência em saúde pública por desastres é imprescindível uma vez que alguns fatores são imprescindíveis, a exemplo de que:

- o conhecimento local é usado para atender às necessidades reais da comunidade;

- a comunidade preparada, ativa e bem organizada pode reduzir os riscos e o impacto das emergências;

- a primeira resposta é essencial pois vidas podem ser salvas nas primeiras horas após o evento por meio de resposta da comunidade antes que a ajuda externa chegue;

- quanto mais preparado o nível local, menos se aciona recursos adicionais.

Para que a saúde da população seja protegida durante e após um desastre, os determinantes mais 
Políticas públicas na gestão de risco de desastres: um olhar para além

da resposta à emergência no Sistema Único de Saúde

amplos da saúde, como a água, o saneamento, a nutrição e a segurança, também precisam ser adequadamente abordados por meio do trabalho multissetorial.

A atuação eficiente do setor envolve, dentre outros aspectos, a articulação interinstitucional, a identificação de serviços prioritários, o estabelecimento de mecanismos de coordenação, a gestão da informação e um processo de comunicação de risco adequado.

\section{CONSIDERAÇÕES FINAIS}

O perfil de saúde de uma população pode ser vista como uma síntese das condições de vida a que está exposta. Aspectos sociais e ambientais estão direta e indiretamente relacionados com o perfil epidemiológico e deles se estabelecem a dimensão dos desafios a serem enfrentados pelo sistema de em situações de desastres.

A atuação oportuna do setor saúde em situações de desastres exige constante análise de situação de saúde e deve estar baseada na prevenção de riscos, incluindo uma abordagem integral sobre o cuidado, planejamento das atividades propostas, avaliação dos impactos gerados, direta ou indiretamente e, ainda, a sensibilidade dos serviços de saúde para identificação de mudanças de comportamento no perfil epidemiológico, durante e após o evento.

A perspectiva de risco deve, ainda, ser avaliada e estar em consonância com o desenvolvimento das políticas públicas de saúde no território, de forma que a atuação do SUS seja efetiva e oportuna, considerando a realidade local e a demanda pelos serviços de saúde.

Nesse contexto, pode-se afirmar que os desastres trazem impactos à saúde e que o fortalecimento de uma agenda de redução do risco desses eventos deve ser parte do processo de trabalho dos diversos setores envolvidos nessa temática (saúde, defesa civil, assistência social, educação, saneamento, etc.) no intuito de reduzir as sobreposições de ações bem como minimizar que a tomada de decisão em uma área possa resultar em risco adicional para outro setor. Esse é um dos principais desafios que se observa hoje na implementação de políticas públicas.

Além disso, o estabelecimento de uma base de evidências científicas para subsidiar a tomada de decisão é necessário para fornecer apoio ao estabelecimento ou fortalecimento de programas multissetoriais e multidisciplinares de gestão de riscos de desastres. Este é outro desafio que se apresenta no momento que o Brasil vive hoje.

\section{REFERÊNCIAS BIBLIOGRÁFICAS}

BARCELLOS, Christovam; QUITÉRIO, Luiz Antônio Dias. Vigilância ambiental em saúde e sua implantação no Sistema Único de Saúde. Revista de Saúde pública, v. 40, p. 170-177, 2006. https://doi.org/10.1590/S0034-89102006000100025

BRASIL. Conselho Nacional de Saúde. Resolução no 588, de 12 de julho de 2018. Institui a Política Nacional de Vigilância em Saúde (PNVS). Brasília, 2018. Disponível em: http://conselho.saude.gov.br/resolucoes/2018/Reso588.pdf

BRASIL. Fundação Nacional de Saúde. Vigilância ambiental em saúde/Fundação Nacional de Saúde. - Brasília: FUNASA, 2002. 42 p.

BRASIL. Ministério da Saúde. Plano Nacional de Saúde e Ambiente no Desenvolvimento Sustentável: Diretrizes para Implementação. Brasília, 1995. Disponível em:

http://bvsms.saude.gov.br/bvs/publicacoes/Planonac.pdf

BRASIL. Ministério da Saúde. Secretaria de Vigilância em Saúde. Departamento de Vigilância em Saúde Ambiental e Saúde do Trabalhador. Plano de Resposta às Emergências em Saúde Pública / Ministério da Saúde, Secretaria de Vigilância em Saúde, Departamento de Vigilância em Saúde Ambiental e Saúde do Trabalhador. - Brasília: Ministério da Saúde, 2014. 44 p. Disponível em: http://bvsms.saude.gov.br/bvs/publicacoes/plano resposta emergencias saude publica.pdf

BRASIL. Ministério do Desenvolvimento Regional. Instrução Normativa n. 2, de 22 de dezembro de 
Políticas públicas na gestão de risco de desastres: um olhar para além da resposta à emergência no Sistema Único de Saúde

2016. Estabelece procedimentos e critérios para a decretação de situação de emergência ou estado de calamidade pública pelos Municípios, Estados e pelo Distrito Federal, e para o reconhecimento federal das situações de anormalidade decretadas pelos entes federativos e da outras providências. Anexo V - COBRADE. Brasília, DF, 2016. Disponível em: http://www.integracao.gov.br.

BRASIL. Presidência da República. Decreto oㅜ 7.616, de 17 de novembro de 2011. Dispõe sobre a declaração de Emergência em Saúde Pública de Importância Nacional - ESPIN e institui a Força Nacional do Sistema Único de Saúde - FN-SUS. Disponível em:

http://www.planalto.gov.br/ccivil 03/ Ato2011-2014/2011/Decreto/D7616.htm

CARUSO GD. The legacy of natural disasters: The intergenerational impact of 100 years of disasters in Latin America. J Dev Econ. 2017. doi:10.1016/j.jdeveco.2017.03.007.

https://doi.org/10.1016/j.jdeveco.2017.03.007

FREITAS, Carlos Machado de (Org.). Agentes locais em desastres naturais: defesa civil e saúde na redução de riscos: livro do aluno. Rio de Janeiro, RJ: Fiocruz, 2014. 169 p.

FREITAS, Carlos Machado de. Guia de preparação e respostas do setor saúde aos desastres / Carlos Machado de Freitas, Maíra Lopes Mazoto e Vânia da Rocha. - Rio de Janeiro, RJ: Fiocruz/Secretaria de Vigilância em Saúde, 2018. 159 p.

OPAS. Organização Pan-Americana da Saúde. Ministério da Saúde. Desastres Naturais e Saúde no Brasil. Brasília, DF: OPAS, Ministério da Saúde, 2015. 56p:. il. (Série Desenvolvimento Sustentável e Saúde, 2).

TAMBELLINI, Anamaria Testa; CÂMARA, Volney de Magalhães. A temática saúde e ambiente no processo de desenvolvimento do campo da saúde coletiva: aspectos históricos, conceituais e metodológicos. Ciência \& Saúde Coletiva, v. 3, p. 47-59, 1998. https://doi.org/10.1590/S141381231998000200005

UGARTE C, AGUILAR P, MAUVERNAY J. Voluntad política , coordinación y planificación : componentes clave para fortalecer la respuesta nacional a emergencias y desastres de salud pública en países de América Latina y. Rev Panam Salud Pública/Pan Am J Public Heal. 2018;42:1-4. http://iris.paho.org/xmlui/handle/123456789/49682. 